\title{
WSN Optimization Algorithm for Traffic Environmental Monitoring
}

\author{
https://doi.org/10.3991/ijoe.v14i12.9495 \\ Li Zhu \\ China Transport Telecommunications \& Information Center, Beijing, China \\ People's Public Security University of China, Beijing, China \\ Jianwu Li $(\bowtie)$ \\ Cyber Space Institute of China Center for Informatica Industry Development, Beijing, China \\ lijw320@163.com
}

\begin{abstract}
In accordance with the features of variety and disparity of traffic information, using wireless sensor network to monitor traffic environment, this paper proposes an optimization algorithm of applying wireless sensor to monitor traffic environment. In this paper, to optimize the coverage, we analyze the irregularity of network sensing areas based on environmental factors and propose a wireless sensor network optimization algorithm. According to the irregularity of network node sensing areas, we build an irregular network coverage model, divide the node monitoring area to improve the network coverage and make corrections to the coverage according to the network coverage connectivity and other features. The simulation test proves that the method proposed in this paper can avoid too many redundant nodes - it only requires a reasonable number of network nodes. This will reduce network energy costs and increase network connectivity without affecting the coverage, thus improving the network performance. the algorithm can satisfy the requirements of real-time acquisition, processing and remote supervision of traffic information.
\end{abstract}

Keywords-traffic information, real-time acquisition, wireless sensor network, sensing radius, environmental factors

\section{Introduction}

Wireless sensor network has certain abilities of aware computing and communication, thus it has been widely used in different areas, such as agriculture, military, medical treatment and transportation, etc. It has the advantages of self-organization and high fault tolerance. However, the wireless sensor node energy is limited, suppressing the network life cycle. If there is a great number of sensor nodes in the network, it will not be convenient to replace batteries [1]. So how to perform the specific monitoring task for as long as possible and achieve monitoring purposes within the effective range is one of the key issues that need to be addressed.

Due to the simple structure, limited energy and great number of sensor nodes, usually the nodes are randomly deployed in the network. Under an ideal condition, it is believed 
that the radius of each node in each direction is the same, i.e. the sensing area is circular. However, wireless sensor networks are often applied in more complex environments, such as monitoring on the growth of agricultural crops and forest environmental monitoring. The changes in these monitoring environments will have some impacts on the communication range between nodes, making the network structure asymmetric, and at the same time, network signals [2-4] are blocked by substances, which makes the sensing areas of nodes irregular and can easily result in monitor dead zones, affecting the accuracy, integrity and timeliness of the overall monitoring information. The random deployment of network nodes cannot guarantee the completeness and effectiveness of network coverage and the coverage quality is poor. In order to ensure that the target monitoring area is within the monitoring range of the wireless sensor network, people need to optimize and control the monitoring range of the target area in a reasonable manner using a certain algorithm, which is a network coverage problem. At present, the wireless network coverage problem is one of the current research hotspots. Slijepcevic et al. proposed a centralized algorithm to calculate the maximum cover set [5]. It does not have good scalability and relies on the location information of the nodes. Charkrabarty et al. used the linear programming method to optimize the nodes in the monitoring area to achieve full coverage [6]. Tian et al. proposed an off-duty eligibility rule, which calculates the covering relations between nodes according to the positions of nodes or receiving angles of signals [7]. The algorithm did not consider the possible overlaps between the node coverage areas, and as a result, there are a great number of work nodes, leading to excessive energy consumption. Reference [8] gave the network node energy consumption conversion model and used this model to reduce network node energy consumption and meet the network coverage requirements. Literature [6] proposes to use a circle to solve node adjustment coverage. It calculates the angle between the awareness circle and the monitored area to get the minimum number of working nodes and reduces redundant working nodes. But under high coverage, the connectivity is weak. Reference [10] proposed an effective coverage algorithm under the awareness model. The algorithm uses the exponential distribution characteristics to calculate the event probability of the sensor node coverage in the monitoring area, then gave the calculation process of the coverage quality based on the event probability and finally achieve the effective coverage of the monitoring area. This algorithm uses the exponential distribution to calculate the coverage quality and achieve the effective coverage of a monitoring area with higher coverage accuracy. However, the above coverage algorithm and model did not take into account the impacts of the irregular sensing areas of the wireless sensor network in actual practice on the coverage quality. Slijepcevic transformed achieving the minimum number of nodes into solving the largest independent node cover set, where there are problems such as the redundancy and absence of the sensing domain. Reference [11] presents an energy-efficient CSSC model that considers energy as a major factor. The sensing range of the model is composed of a circular sector with a variable central angle and a variable sensing radius, depending on the residual energy information exchange between nodes, but the model does not make corrections to the coverage according to the network coverage connectivity and other features. In this paper, we intend to analyze the irregularity of network sensing radius. Based on the irregularity of the node sensing areas in the network, we build an 
irregular sensor model and proposes a coverage strategy for the wireless sensor network based on irregular sensing areas, which divides the node monitoring area to improve the network coverage and makes corrections to the coverage according to the network coverage connectivity and other features. This strategy can minimize the redundant coverage areas and dead zones in the network, reduce the overall energy consumption of the network and increase network connectivity.

\section{Network model and problem description}

\subsection{Network Model}

Supposing a node set $\mathrm{S}$ which containing $\mathrm{N}$ non-overlapping wireless sensor nodes is randomly deployed in a 2-D monitoring region A. After deployment, the position of each node is fixed. All nodes have a perception radius of $R_{S}$ and a communication radius of $R_{c}$, where $R_{s} \geq 2 R_{c}$. To any node $S_{i}\left(S_{i} \in S\right)$, as the center of a circular perception area. Supposing the node has the following properties:

1. The wireless sensor nodes are randomly deployed in the monitoring region. After deployment the node location is fixed, and a wireless Ad-Hoc network is building up.

2. The node's communication radius is two times as perception radius, that is $R_{s}=2 R_{c}$. Any two nodes located within communication radius are neighbor nodes to each other.

3. The node perception coverage area is $\xi=\pi \mathrm{R}_{\mathrm{s}}^{2}$. If and only if any node $\mathrm{p}$ in the monitoring region satisfies the rule $\left\|S_{i} \bullet p\right\| \leq R_{s}$, it is called that node $p$ is covered by $\mathrm{Si}$.

4. The accurate location of fixed node is known.

5. All sensor node has an initial energy $\mathrm{W}$, and they have synchronous clock.

When studying the coverage characteristics of irregular sensing areas in a wireless network, we consider the characteristics of the network system and application requirements and mainly look at the following two indicators.

Effective coverage rate: the standard definition is the ratio between the total area covered by all the nodes and the total target area. In order to evaluate the effective coverage rate of the network, we perform the edge detection and analysis on the node sensing domain and calculate the total effective coverage area.

Coverage uniformity: the coverage uniformity represents the equilibrium energy load capacity of the network and reflects the network redundancy. We use the standard deviation of the inter-node distances to present the coverage uniformity. The smaller the standard deviation is, the better the coverage uniformity will be, and the fewer overlapping sensing areas there will be.

The sensor nodes in the network are deployed in the monitoring area $\mathrm{M}$ in a random and uniform manner. If all nodes are in service, they can cover the whole area and ensure the network connectivity. Now we assume this wireless sensor network has the 
following properties: (1) Compared with the node sensing area, the monitoring area $\mathrm{M}$ is big enough and the border factors are negligible; (2) The locations of all sensor nodes are fixed and can be acquired by the existing localization methods; (3) All nodes are in the same status in the network and performance parameters like initial energy are the same.

For most sensing signals, when a wireless signal encounters [12-15] any obstacle in the transmission process, it will change the received signal power, which may lead to signal blocking or loss and also differences in the signals received by a node in different directions. As a result, the wireless sensor network is irregular during the propagation process. When the sensing range of a sensor node is a regular circle [16], the relationship between connectivity and coverage [17-18] under the condition where the communication radius is greater than the sensing radius is that: when the communication radius is greater than or equal to twice the sensing radius, as long as the area is fully covered, full connectivity will be ensured. However, a sensor node has different sensing capabilities in different directions, forming an irregular sensing range. Therefore, in this paper, we will discuss the connectivity and coverage issues in an irregular sensing model.

The effective coverage rate of a wireless sensor network is closely related to the sensing ranges of nodes in the monitoring area. In the wireless signal transmission process, the signal strength decreases with the increase in the signal transmission distance. However, in the process of signal transmission, obstacles make the sensing range of a network node irregular. Ideally, the propagation loss ratio will decrease as the signal transmission distance increases.

When there are $K$ sensor nodes in a monitoring area, the coverage probability of a sensor node is defined as the probability of a point being sensed by at least one of the $K$ sensor nodes. Supposing there is a random point a in the monitoring area $A$, if this point can be sensed by a sensor node $S$ around it, this point is an effectively sensed point, and it can receive wireless signals correctly. In the node sensing process, let $W_{k}$ be the path loss intensity threshold. If the inter-node path loss intensity is lower than $W_{k}$, it means this point cannot be sensed by any sensor node and thus it cannot effectively transmit wireless signals. This position is an ineffective node location, i.e. monitoring blind spot. In order to determine the effective sensing area of a random sensor node a in the monitoring area, we assume the probability of a random node $b$ in the monitoring area being effectively sensed by the sensor $\mathrm{a}$ is $\mathrm{p}(\mathrm{a})$, and $\mathrm{n}$ number of nodes $b$ form the effective sensing area of $a$. As it is not a regular circle under the ideal condition, the effective sensing area $S$ is calculated.

\subsection{Definition and description}

Definition 1: (Coverage Set) Monitoring region A is divided into different Voronoi polygon regions, and sensor node set $\mathrm{S}$ is deployed in these regions, that is $S_{i} \in V_{i}(S, R, V)$, where $V_{i}$ is the Voronoi polygon region $S_{i}$ located. Sensor node set $\mathrm{D} \subseteq \mathrm{S}$, if the perception region of the nodes within $\mathrm{D}$ can completely cover the monitoring region, $\mathrm{D}$ is called a coverage set. 
Definition 2: (Effective Coverage Region) In any Voronoi polygon, if the node $\mathrm{i}$ perceives the existence of the target node $\mathrm{k}$ when $\mathrm{k}$ appears, then $\mathrm{k}$ is covered by $\mathrm{i}$. If all nodes in a certain region are covered by $i$, this region $\Psi(i)$ is called the coverage region of node $i$. Thus effective coverage region of node $i$ is the intersection between coverage region $\Psi(\mathrm{i})$ and the target region $\Omega$ or target node $\mathrm{k}$. The expression effective coverage region is shown as below.

$$
\zeta(\mathrm{i})=\Psi(\mathrm{i}) \cap \Omega
$$

Definition 3: (Coverage Rate) Coverage rate $\eta$ is the ratio of the effective coverage region to the monitoring region of all the operation nodes in monitoring region $\mathrm{A}$ at a certain time. It is an important indicator of network coverage performance measurement.

$$
\eta=\frac{\sum_{i=1}^{n} \xi(i)}{A}
$$

Definition 4: (Energy balance factor) According to the regional energy value, the energy within the domain is quantified and evaluated. The ratio between the regional energy maximum value and minimum value and the regional energy mean value in the domain is defined as the energy balance factor:

$$
\rho=\frac{Q_{\mathrm{k} \max }-Q_{\mathrm{k} \text { min }}}{\frac{1}{\mathrm{k}} \sum_{\mathrm{k}=1}^{\mathrm{n}_{\mathrm{i}}} \mathrm{Q}_{\mathrm{ik}}}
$$

$\rho$ can reflect the balanced distribution of node energy in different domains throughout the network. When the value of $\rho$ is larger, the distribution of energy consumption in the whole domain is less balanced. When the value of $\rho$ is the smaller, the more balanced the overall energy distribution is in the entire domain.

\section{$3 \quad$ Optimization strategy}

To cover the irregular areas, the first step is always to prepare the target area, because this step will directly affect the network coverage effects. Facing the complex nonlinear boundaries of the target area, firstly, we should calculate the number of neighbour nodes to a given node and approximate the target area into a polygon area; secondly, we need to build a minimum binding area of the target area; finally, we need to discretize the target area by marking the areas that are not part of the target area and not needed in the coverage task in the rectangle as redundant coverage areas and removing the corresponding geometric center from the nodes; as the geometric center of some grid is not inside the polygon, in order to ensure full coverage of the target area, we need to adjust the minimum area. At last, we obtain a quasi-optimal cover set $C$. 
Supposing the minimum number of neighbour nodes required to make the probability of a node sensing area being covered by neighbour nodes no less than the specified value is $\mathrm{m}$, that the area of the whole network is $S$, and that the sensing area of a single node $i$ in the network is $S_{i}$, the probability $p$ of a random point in the network being covered by the node $i$ is $p=\frac{S_{i}}{S}$.

If there are $i$ nodes in the network, as the nodes in the network all have the same parameters, the probability $D$ of any of the $i$ nodes covering a random point in the network is the same, and the probability $Q$ of a random point in the network not being covered by any of the $i$ nodes. Then the probability $E_{k}$ of a random point in the network being covered by at least one of the $k$ nodes i.

Considering that there exists a point in the sensing domain of any node $S_{i}$ in the optimal cover set $C$ that is sensed by at least one neighbour node, the overlap between the sensing areas of the neighbour node $v$ and $S_{i}$ meet $E(v)<E_{0}$, and the percentage of the sensing area of the node $S_{i}$ covered by the neighbour node is no less than $\eta_{0}$, we use the fuzzy optimal solution method to obtain the number of nodes in the optimal coverage set $C$.

Network connectivity is one of the key factors to measure the network performance. Considering that the coverage and connectivity of the network is correlated to some extent, we build a connected cover set in the network to make the network coverage and connectivity balanced and optimize the overall performance of the network. The nodes in the network coverage can guarantee good coverage, but the nodes may not be interconnected. Supposing that N sensor nodes are randomly deployed in the monitoring area $\mathrm{A}$, based on the optimal node selection method for irregular sensing areas, we obtain the optimal cover set $\mathrm{C}$ in the network. And based on the properties of each node in the optimal cover set $\mathrm{C}$ in the network and the irregularity of their sensing areas, we build the minimum connected cover set $\mathrm{D}$ in the network.

We choose a random point $S_{i}$ from the cover set $C$, i.e. $S_{i} \in C$. The neighbour node set of $\mathrm{Si}$ is $\mathrm{M}$. We choose a random point $S_{j}$ from $M$, and $i \neq j$. According to the relationship factor for the distance between neighbour nodes in the network and the irregular sensing area $\Gamma=\sum_{\mathrm{i}=1}^{\mathrm{n}} \mathrm{f}\left(\mathrm{M}, \mathrm{d}(\mathrm{i}, \mathrm{j}), \Phi\left(\mathrm{P}_{\mathrm{ij}}, \mathrm{R}_{\mathrm{s}}\right)\right)$, with $S_{i}$ as the center, and the effective boundaries of the irregular sensing area as the boundaries, the node $S_{i}$ sends its own information such as radius, node energy and number of neighbour nodes to its neighbour nodes in different directions to determine the connected domain of the neighbour nodes $\Omega_{i}$. And similarly, the connected domain of the neighbour nodes of all nodes in the cover set $C$ is $\Omega$. We choose a random node in the cover set $C S_{i}$, obtain the coverage of the connected domain of neighbour nodes $\Omega$ and its sensing area, and convert it into a weighted connected graph of irregular boundaries and sub-coverage domains. If for the connected subgraph of $V\left(S_{i}\right)$, the coverage is not less than the coverage threshold $\eta$, we add $\mathrm{Si}$ and the fixed point $V_{j}$ of its connected subgraph to the connected cover set E. otherwise, we only add $\mathrm{Si}$ to the connected cover set $E$. We rebuild this connected cover set $\mathrm{E}$ for $\mathrm{m}$ times until we traverse all the points in the cover set $C$. At last, we build a non-overlapping minimum connected cover set $D$ and $C \cap \Omega=\Phi$. 
In the network operation process, there are dead zones and redundant coverage areas, so we need to make connectivity-based corrections to these coverage areas to improve the overall network coverage performance. We approximate the area of the target zone. These target zones can be classified into dead zones and redundant areas. If this area is a dead zone, we obtain the area boundary region and solve the approximate area $M$ of $A i$. By using the area weighting function, we obtain the weight of this area, i.e. $\theta_{\mathrm{i}}=\mathfrak{R}\left(\mathrm{M}_{\mathrm{i}}, \partial \mathrm{i}\right)$, where $\partial \mathrm{i}$ is the connectivity of the central node of the area and its neighbor nodes. Then we obtain the weight set $\Theta$ of the dead zones in the monitoring area; and similarly, the weight set $\rho$ of the redundant area.

If the repair area is the dead zone $A_{i}$, the distance from any point ai in the irregular boundary region with $\mathrm{H}$ as the approximate center to $\mathrm{H}$ is $\mathrm{L}_{\mathrm{i}} \in\left[\mathrm{L}_{\min }, \mathrm{L}_{\max }\right]$ ). $\alpha_{0}$ is the coverage threshold angle, and $\alpha_{0}=\frac{\hat{\Theta}}{2}$, where $\hat{\Theta}$ is the coverage angle formed by the maximum and the minimum boundary points in the boundary region, and $\hat{\Theta}<\pi$. If we choose a node $K_{i}$ which is the nearest to the node $\mathrm{H}$ from the minimum connected cover set $\mathrm{D}$, then $\mathrm{M}\left(\mathrm{a}_{\text {min }}, \mathrm{a}_{\text {max }}\right)=\left\{\mathrm{k}_{\mathrm{i}} \in \mathrm{R}^{2} \mid \mathrm{d}\left(\mathrm{k}_{\mathrm{i}}, \mathrm{H}\right) \leq \mathrm{d}\left(\mathrm{k}_{\mathrm{j}}, \mathrm{H}\right)\right\}$, where $\mathrm{M}\left(\mathrm{a}_{\left.\min , \mathrm{a}_{\max }\right)}\right.$ stands for $K_{i}$, the node in the area $A_{i}$ nearest to $\mathrm{H}$. In order to ensure network connectivity during the correction of the dead zone, with $K_{i}$ as the reference point, we choose a node $K_{j}$ which is the nearest to $K_{i}$ in the minimum connected cover set $D$ and form the minimum coverage angle $\varphi=\angle \mathrm{K}_{\mathrm{i}} H \mathrm{~K}_{\mathrm{j}}$ in this repair area. If $\varphi \in\left(0, \alpha_{0}\right)$, then $K_{i}$ is the repair node of this dead zone; if $\varphi \in\left(0, \alpha_{0}\right)$, with the angular bisector $\mathrm{Li}$ of $\varphi$ as the boundary, we choose a node $K_{m}$ which is the nearest to Li from the minimum connected cover set $D$. In this case, the nodes $K_{i}$ and $\mathrm{km}$ are the repair nodes of this dead zone; if $\varphi \in(\pi, 2 \pi)$, we divide $\varphi$ by half, with $\alpha_{0}$ as the critical value, until the angle divided $\varphi_{i} \leq \alpha$. The selection method for repair nodes is as above. If the repair area is a redundant area, we select the redundant area Bi according to the weight set $\rho$ for the redundant area. The approximate center of the irregular boundary region is ci, and the redundant area of $\mathrm{Bi}$ is $\mathrm{M}_{\mathrm{i}}\left(\mathrm{r}_{\mathrm{i}}\right)=\sum_{\mathrm{i}=1}^{\mathrm{k}} \pi \Gamma\left(\mathrm{r}_{\mathrm{i}}^{2}, \mathrm{t}\right) . S_{i}$ is the area of each redundant subarea in $B_{i}$, and $l$ is the redundancy coefficient of the redundant subarea. Based on the existing network connectivity, and considering the irregularity of the sensing area, we adjust the sensing radius to make minimum repair to the redundant area. According to the linear weighting and the effectiveness of sensing distance, we know that the redundant areas in the domain are convergent, and thus there exists a minimum value. Therefore, we can obtain the quasi-optimal solution of the sensing radius $r_{i}^{*}$, which makes the redundant area function $M_{i}$ reach the minimum value, i.e. $r_{i}^{*}=\operatorname{argr} r_{i} \in R \min M_{i}\left(r_{i}\right)$. By analyzing the minimum value of the redundant area, we use the equilibrium between multiple connected nodes in the same area $\mathrm{B}_{\mathrm{i}}$ to obtain the quasi-optimal solution to the sensing radius, and in this way minimize the redundant coverage area.

Conclusion 1: when the nodes are randomly deployed, in the coverage model based on irregular sensing areas, the minimum number of nodes to achieve the effective coverage of the monitoring area should satisfy the following condition: 


$$
n>\tau \ln \left(1-\frac{\Theta \pi\left(\int_{0}^{2 r} x \frac{1}{2 r} d x\right)^{2}}{d^{2}}\right)^{-1}
$$

Proof: let's suppose in the coverage area, the coverage rate of the sensor nodes is $P$. In the coverage area, when the sensor nodes are in service, they are all correlated. Therefore, the probability of two sensor nodes being in service is:

$$
p\left(s_{1}+s_{2}\right)=1-(1-p)^{2}
$$

the coverage rate of point $i$ in the coverage area is:

$$
p(i)=1-\prod_{1<i<k}(1-p)
$$

For any sensor node with non-zero energy, the coverage rate is greater than $\varepsilon$, where $\varepsilon$ is the given infinitely-small positive variable

$$
1-\prod_{1<i<k}(1-p)>\varepsilon
$$

at the same time $(1-p)^{n}>\varepsilon$. For any point $i$ in the monitoring area $\Omega$, the cover probability is:

$$
p_{i}=\int_{0}^{2 r} \kappa \frac{\theta \pi \Psi(r)}{2 r} e^{-\frac{\left(r-r_{0}\right)^{2}}{2 \sigma^{2}}} d x
$$

From this, we can see that $\mathrm{n}$ is the minimum number of sensor nodes in the network:

$$
n>\tau \ln \left(1-\frac{\Theta \pi\left(\int_{0}^{2 r} x \frac{1}{2 r} d x\right)^{2}}{d^{2}}\right)^{-1}
$$

\section{$4 \quad$ Test and analysis}

Through the simulation test, we compare the performances of the algorithms. The test scenario is set up as follows: set up 120 sensor nodes with a sensing radius of 5$20 \mathrm{~m}$ in a monitoring area with a size of $1000 \times 1000 \mathrm{~m}^{2}$, and the initial energy of each node is $200 \mathrm{~J}$. We compare the algorithm proposed in this paper (TMOA) and the existing algorithm random and mainly analyze the following performances. 


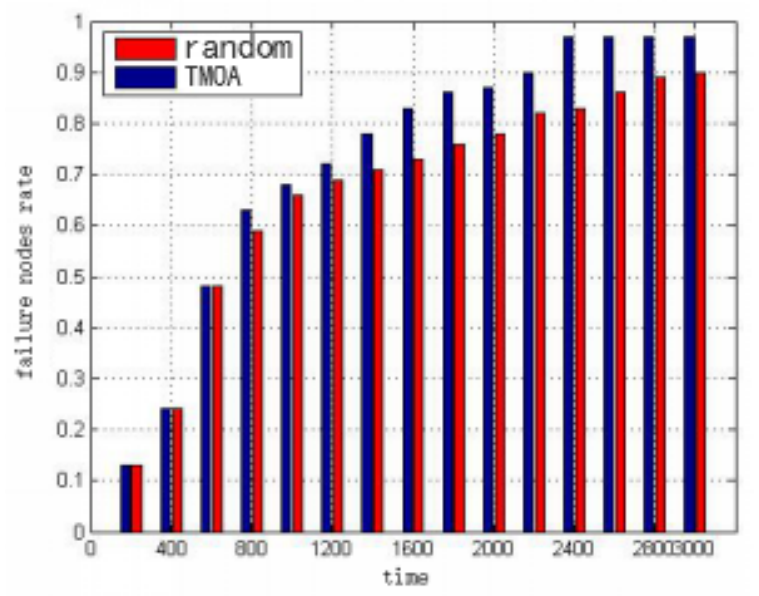

Fig. 1. The failure nodes

In the network operation process, "failure" nodes will undoubtedly bring great impacts on the network performance, including network connectivity, energy consumption and lifetime, etc. We use the network node failure rate to evaluate the "failure" nodes. The network node failure rate is the ratio between the number of "failure" nodes and the total number of nodes, which is a performance index that can directly reflects the running status of the network nodes. It can be seen from Fig. 1 that the number of failure nodes in TMOA algorithm is relatively stable at the later stage of network operation, the node failure ratio changes slower than that in the random algorithm and there are fewer failure nodes than in the random algorithm. This further indicates that the TMOA algorithm can minimize the number of failure nodes, reduce network overhead, and extend the node service time.

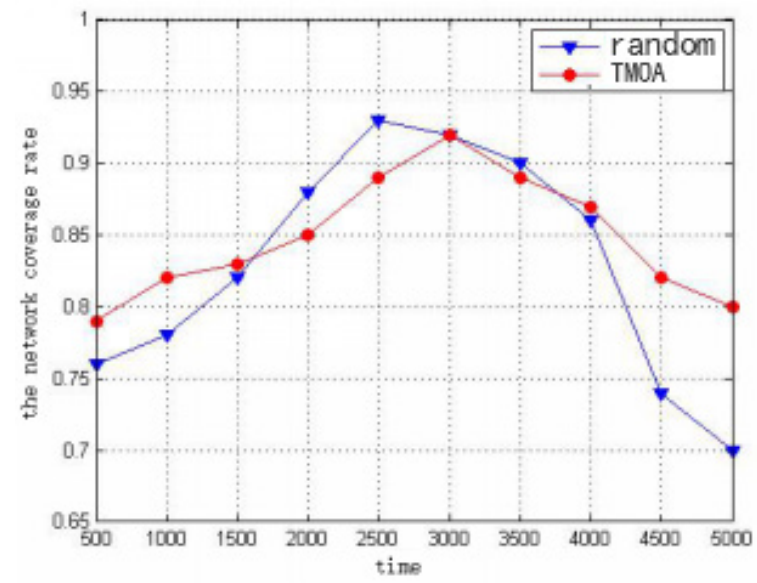

Fig. 2. The network coverage performance 
The network lifetime is one of the important indicators to measure the network performance. The TMOA algorithm proposed in this paper takes into account the impact of the residual energy of nodes on the network lifetime. By associating the remaining energy of nodes with the sensing range, it adjusts the sensing range according to the electric quantity and the coverage rate, which effectively extends the lifetime of the nodes with a little amount of electricity left. From Fig. 2, we can see that when the network coverage rate is similar, the network lifetime in the TMOA algorithm is significantly longer than that in the random algorithm, and this advantage is more obvious over time. We test the network connectivity in the two algorithms in the same parameter setting and find that the approximate minimum cover set obtained by the algorithm proposed in this paper based on the minimum connected cover set contains fewer nodes. the coverage of the approximate minimum cover set adopted by the TMOA algorithm brings higher data transmission rate with the increasing number of nodes. This coverage enhancement method improves the connectivity of the network coverage area. When the network connectivity is poor, the coverage enhancement method can improve the connectivity significantly.

\section{Conclusions}

In a wireless sensor network, the network coverage rate and connectivity are important factors that can affect the control performance of network coverage. In this paper, we propose a wireless sensor network coverage strategy based on irregular sensing areas. Based on the irregularity of network node sensing areas, we build an irregular network coverage model, divide the node monitoring area to improve the network coverage rate and make corrections to the coverage according to the network coverage connectivity and other features. This strategy can reduce the redundant coverage areas and dead zones in the network and thus reduce the overall energy consumption of the network. The test results show that the method proposed in this paper can avoid too many redundant nodes - it only requires a reasonable number of network nodes in the network, which will reduce network energy costs and increase network connectivity, and thus improve the network performance.

\section{Acknowledgments}

This work was supported by key special project of National Key Research and Development Program (2017YFC0803900).

\section{$7 \quad$ References}

[1] Yang, S, Dai, F, Cardei, M, Wu, J., Patterson F. (2006). On connected multiple point coverage in wireless sensor networks. Wireless Information Networks, 13(4), pp.289-301. https://doi.org/10.1007/s10776-006-0036-Z 
Paper-WSN Optimization Algorithm for Traffic Environmental Monitoring

[2] Xing, X.F., Wang, G.J., Li J. (2015). Polytype target coverage scheme for heterogene-ous wireless sensor network using linear programming. Wireless Communications and Mobile Computing, 14, pp.1397-1408. https://doi.org/10.1002/wcm.2269

[3] Mini, S., Udgata S.K., Sabat S.L. (2014). Sensor deployment and scheduling for target coverage problem in wireless sensor networks. IEEE Sensors Journal, 14(3), pp.636-644. https://doi.org/10.1109/JSEN.2013.2286332

[4] Jing, H.C. (2014). Coverage holes recovery algorithm based on nodes balance distance of underwater wireless sensor network. International Journal on Smart Sensing and Intelligent Systems, 7(4), pp. 1890-1907. https://doi.org/10.21307/ijssis-2017-738

[5] Slijepcevic, S, Potkonjak, M. (2001). Power efficient organization of wireless sensor networks. IEEE International Conference on Communications, 2, pp.472-476. https://doi.org/10.1109/ICC.2001.936985

[6] Chakrabarty, K., Lyengar, S.S., Qi, H., Cho, E. (2002). Grid coverage for surveillance and target location in distributed sensor networks. IEEE Trans. on Computers, 51(12), pp.14481453. https://doi.org/10.1109/TC.2002.1146711

[7] Tian, D., Georganas, N.D. (2003). A node scheduling scheme for energy conservation in large wireless sensor networks. Wireless Communications and Mobile Computing, 3(2), pp.271-290. https://doi.org/10.1002/wcm.116

[8] Hefeeda, M., Bagheri, M. (2007). Randomized k-coverage algorithms for dense sensor networks. Proceedings of IEEE International Conference on Computer Communica-tions, 612, pp.2376-2380. https://doi.org/10.1109/INFCOM.2007.284

[9] Khedr, A.M., Osamy, W. (2011). Minimum perimeter coverage of query regions in a heterogeneous wireless sensor network. Information Sciences, 181(15), pp. 3130-3142. https://doi.org/10.1016/j.ins.2011.04.008

[10] Tan, R., Xing, G.L., Liu, B.Y., Wang, J.P., Jia, X.H. (2012). Exploiting data fusion to improve the coverage of wireless sensor networks. IEEE/ACM Trans. on Networking, 20(2), pp.450-462. https://doi.org/10.1109/TNET.2011.2164620

[11] Soreanu, P., Volkovich, Z. (2009). Energy-Efficient Circular Sector Sensing Coverage Model for Wireless Sensor Networks. The 3rd International Conference on Sensor Technologies and Application (SENSORCOMM), 18-23, pp. 229-233. https://doi.org/10.1109/SEN SORCOMM.2009.45

[12] Halber, A., Chakravarty, D. (2018). Investigation of wireless tracking performance in the tunnel-like environment with particle filter, Mathematical Modelling of Engi-neering Problems, 5(2), 93-101. DOI: 10.18280/mmep.050206 https://doi.org/10.18280/mmep.050206

[13] Bucur, A., Achimaş, G., Lăzărescu, L. (2017). Evaluation of environmentally friendly lubricants by ring compression test. Academic Journal of Manufacturing Engineer-ing, 15(2), pp. 37-42.

[14] Zhang, J., Varadharajan, V. (2010). Wireless sensor network key management survey and taxonomy. Journal of Network and Computer Applications, 33(2), pp. 63-75. https://doi.org/10.1016/i.jnca.2009.10.001

[15] Li, H.J., Xu, G.N., Zhou, L.D. (2017). Mechanical parameters acquisition test based on wireless sensor network, Academic Journal of Manufacturing Engineering, 15(4), 88-93.

[16] Erdelj, M., Razafindralambo, T., Simplot-Ryl, D. (2013). Covering points of interest with mobile sensors. IEEE Trans. on Parallel and Distributed Systems, 24(1), pp.32-43. https://doi.org/10.1109/TPDS.2012.46

[17] Sun, Z.Y., Wu, W.G., Wang, H.Z., Chen, H., Wei, W. (2014). An optimized strategy coverage control algorithm for WSN. Int'l Journal of Distributed Sensor Network, 11, pp.1-12. https://doi.org/10.1155/2014/976307 
[18] Li, Y., Wei, D.D., Mu, Z., Xiong, Z.H., Wang, Y.H., Yin, W.S. (2015). Study of the timecollocation of signal lamp at intersection. Mathematical Modelling Of Engi-neering Problems, 2(2), pp. 5-10 https://doi.org/10.18280/mmep.020202

\section{Authors}

Li Zhu, received her M.S. degree in Computer Applied Technology from University of Electronic Science and Technology of China (2011). She received her doctor degree at Beijing University of Posts and Telecommunications (2017). Her research interests include WSN and embedded system. Email: zhul_jolie@163.com

Jianwu Li, Senior Engineer Doctor of engineering, Graduated from Beijing University of post and telecommunications in 2015. Currently Deputy Director of Mobile Internet Applications. Email: lijw320@163.com

Article submitted 04 September 2018. Resubmitted 28 September 2018. Final acceptance 14 October 2018. Final version published as submitted by the authors. 